\title{
Functionalisation of graphene surfaces with downstream plasma treatments
}

\author{
Niall McEvoy ${ }^{a}$, Hugo Nolan $^{a, b}$, Nanjundan Ashok Kumar ${ }^{a}$, Toby Hallam ${ }^{a}$, \\ Georg S. Duesberg ${ }^{a, b, *}$ \\ a CRANN, Trinity College Dublin, Dublin 2, Ireland \\ ${ }^{\mathrm{b}}$ School of Chemistry, Trinity College Dublin, Dublin 2, Ireland
}

\begin{abstract}
We report on an adjustable process for the functionalisation of graphene surfaces with a downstream plasma source. The parameters of oxygen plasma treatments are modified such that oxygenated functionalities can be added to the surface of graphene films prepared by chemical vapour deposition in a controlled manner. The nature of induced defects is investigated thoroughly using Raman and X-ray photoelectron spectroscopy. A massive change in the surface properties is observed through the use of contact angle and electrochemical measurements. We propose the usage of such plasma treatments to facilitate the addition of further functional groups to the surface of graphene. The incorporation of nitrogen into the graphene lattice by substitution of oxygenated functional groups is demonstrated outlining the validity of this approach for further functionalisation.
\end{abstract}

\section{Introduction}

Graphene has garnered much interest from the research community due to its unique physical, structural and electronic properties [1-3]. Recent advances in chemical vapour deposition (CVD) growth of graphene have led to a massive improvement in its processing and scalability [4,5]. Graphene has been linked with applications in next generation electronics [6-10], sensors [11-13] and energy storage and conversion [14-17] to name but a few. Central to the realisation of these applications is control over the quality and surface chemistry of graphene. Plasma treatments allow for the introduction of controlled levels of functionalities onto surfaces without the need for wet chemical steps. This makes it a clean, green technique, compatible with industrial processes. Plasma treatments have previously been used for the modification of polymer surfaces [18] as well as functionalisation of carbon nanotubes [19-21] and pyrolytic carbon [22,23]. These treat- ments can be applied in situ to surface bound graphene and 3D arrangements and have been deemed useful in graphene processing for removing polymer residue [24], thinning multilayer samples [25] and etching edges [26]. A number of groups have also used plasma treatments to functionalise graphene surfaces. Such plasma treatments break the perfect $\mathrm{sp}^{2}$ lattice of the graphene crystal and introduce different functional groups on the surface, thus modifying the surface chemistry and the electronic structure. Gokus et al. exposed a micromechanically exfoliated piece of graphene to $\mathrm{a}_{2} \mathrm{RF}$ plasma and subsequently demonstrated photoluminescence [27]. N-doping of mechanically exfoliated monolayer and CVD bilayer graphene through the use of $\mathrm{NH}_{3}$ plasma treatment was reported by Lin et al. [28]. Conversion of graphene to graphane through the use of a $\mathrm{H}_{2}$ DC plasma was outlined by Elias et al. [29]. Xie et al. fabricated an actuator from a film of liquid phase exfoliated graphene by treating one side of it with hexane and the other side with an $\mathrm{O}_{2}$ plasma [30]. Recently, Liu 
et al. reported the controlled oxidation of graphene using a water vapour plasma [31]. A water vapour plasma was used as the oxidiser with a nanosphere lithography mask so that oxidation would proceed in a milder and more controllable manner.

In this paper we report on the use of a downstream oxygen plasma for the functionalisation of CVD graphene surfaces. In this scenario the samples are placed downstream from the plasma source so that generated ions are relaxed upon arrival at the graphene surface and functionalise it in a controllable manner. This can be considered a chemical plasma as, whilst the radicals are kinetically relaxed, they are still chemically active. This is performed without any form of physical masking and is seen to modify the surface properties without physically damaging the films; that is to say, no perforations, holes or tears are observed in samples following treatment. Scanning Raman spectroscopy gives us insight into the nature and placement of defects on the graphene surface and allows for these to be visualised over a large area. X-ray photoelectron spectroscopy (XPS) allows for the induced defects to be linked with specific oxygenated functionalities. The addition of these oxygenated functionalities could be used as a stepping stone for the addition of controlled levels of larger functional groups which bind preferentially to specific sites. This is demonstrated through the use of a subsequent ammonia plasma treatment which simultaneously removes oxygen groups and introduces nitrogen into the graphene lattice.

\section{Experimental setup}

\subsection{Graphene growth}

Graphene was grown on $\mathrm{Cu}$ foil (Gould, $25 \mu \mathrm{m}$ ) using a process similar to that described elsewhere previously $[5,32]$. Briefly, samples were introduced into a Carbolite tube furnace and ramped to $1035^{\circ} \mathrm{C}$ under $\mathrm{H}_{2}$ flow $(80 \mathrm{sccm}$, Pressure $\sim 0.2$ Torr). The $\mathrm{Cu}$ was annealed for $20 \mathrm{~min}$ at this temperature to remove any oxides and increase the $\mathrm{Cu}$ grain size. The growth step entailed a mixture of $\mathrm{CH}_{4}(10 \mathrm{sccm})$ and $\mathrm{H}_{2}(2.5 \mathrm{sccm})$ for $20 \mathrm{~min}$ (Pressure 0.07 Torr) after which the $\mathrm{CH}_{4}$ flow was switched off and the samples cooled to room temperature under $\mathrm{H}_{2}$ flow. Graphene was transferred to $\mathrm{SiO}_{2}$ on $\mathrm{Si}$ (100) substrates using the established PMMA supported transfer technique which has previously been demonstrated on a large scale [4].

\subsection{Plasma treatment}

Plasma treatments were performed using a $R^{3} T$ TWR-2000T microwave radical generator. Typical experimental conditions involved an output power of $1 \mathrm{~kW}$, an $\mathrm{O}_{2}$ flow rate of $100 \mathrm{sccm}$, exposure times in the range $10-300 \mathrm{~s}$ and a chamber pressure of 1 Torr. Samples were positioned $\sim 30 \mathrm{~cm}$ downstream from the plasma source. In this position the plasma generated ions were energetically relaxed upon arrival at the sample and thus the plasma could be considered remote, minimising surface damage. Reducing plasma treatment was carried out at with a mixture of $\mathrm{H}_{2}$ and $\mathrm{NH}_{3}$ gas ( $50 \mathrm{sccm}$ flow rate for each) for $30 \mathrm{~min}$ at a power of $500 \mathrm{~W}$.

\subsection{Spectroscopic analysis}

Raman measurements were performed using a Witec Alpha $300 \mathrm{R}$ with a $532 \mathrm{~nm}$ excitation laser. Raman spectra were taken of graphene samples transferred to $\mathrm{SiO}_{2}$ substrates. Average spectra were generated from 6400 point scans. Raman maps were generated by taking scans every $250 \mathrm{~nm}$ in the $x$ and $y$ direction, typically over areas of $30 \times 30 \mu \mathrm{m}(120 \times 120$ spectra).

XPS analysis was performed using an Omicron ESCA system with an EA 125 Analyser and XM1000MK II monochromatic X-ray source. The $\mathrm{Al} \mathrm{K} \alpha \mathrm{X}$-ray line was used with a spot size of $\sim 2 \mathrm{~mm}$. The analyser was operated with a pass energy of $50 \mathrm{eV}$. For wide scans a step size of $0.2 \mathrm{eV}$ was employed, while fine scans had a step size of $0.1 \mathrm{eV}$.

XPS was carried out on as-grown and plasma treated graphene films on $\mathrm{Cu}$ foil. Performing measurements on $\mathrm{Cu}$ foil without transferring to $\mathrm{SiO}_{2}$ substrates ensures that the sample is very conductive and is not affected by charging during the measurements; it also reduces the likelihood of impurities or defects being introduced during the transfer procedure.

\subsection{Electrochemical analysis}

Electrochemical analysis was performed using a Gamry Ref 600 potentiostat with a three electrode configuration. Platinum wire and $\mathrm{Ag} / \mathrm{AgCl}$ were used as counter and reference electrodes, respectively. Graphene films were mounted into a plate material testing cell whereby a nitrile $O$ ring defined the region of the electrode exposed to the electrolyte (radius $=5 \mathrm{~mm}$ ) and $50 \mathrm{~nm}$ of gold was used to contact the periphery of the film.

\section{Results and discussion}

Downstream plasma sources generate a high density of ionised species; however these are not attracted to the substrate due to the absence of an applied bias. It is predominantly radicals which reach the surface and react with the $\mathrm{sp}^{2}$ lattice of the graphene crystal. In the case of oxygen plasma this introduces different oxygenated groups to the surface, thus modifying the surface chemistry and the electronic structure.

The effect of our plasma treatments is most immediately obvious by looking at contact angle measurements using a $\mu \mathrm{L}$ water droplet as shown in Fig. 1. The as-grown graphene on $\mathrm{SiO}_{2}$ sample has a measured contact angle of $93^{\circ}$ making it hydrophobic and comparable with previously reported values for graphene [33]. Following plasma treatment, the contact angle is greatly reduced; it is seen to be $31^{\circ}$ for a $10 \mathrm{~s}$ treatment and $26^{\circ}$ for a $300 \mathrm{~s}$ treatment, respectively (standard conditions, $\mathrm{P}=1000 \mathrm{~W}, \mathrm{O}_{2}$ flow $=$ $100 \mathrm{sccm}$ ). This markedly increased hydrophilicity can be attributed to the affinity of water molecules for oxygenated functionalities introduced on the graphene surface. No obvious physical deterioration of the film is observed using optical techniques. Furthermore, the contact angle post treatment is considerably smaller than that of the underlying substrate $\left(56^{\circ}\right)$. 


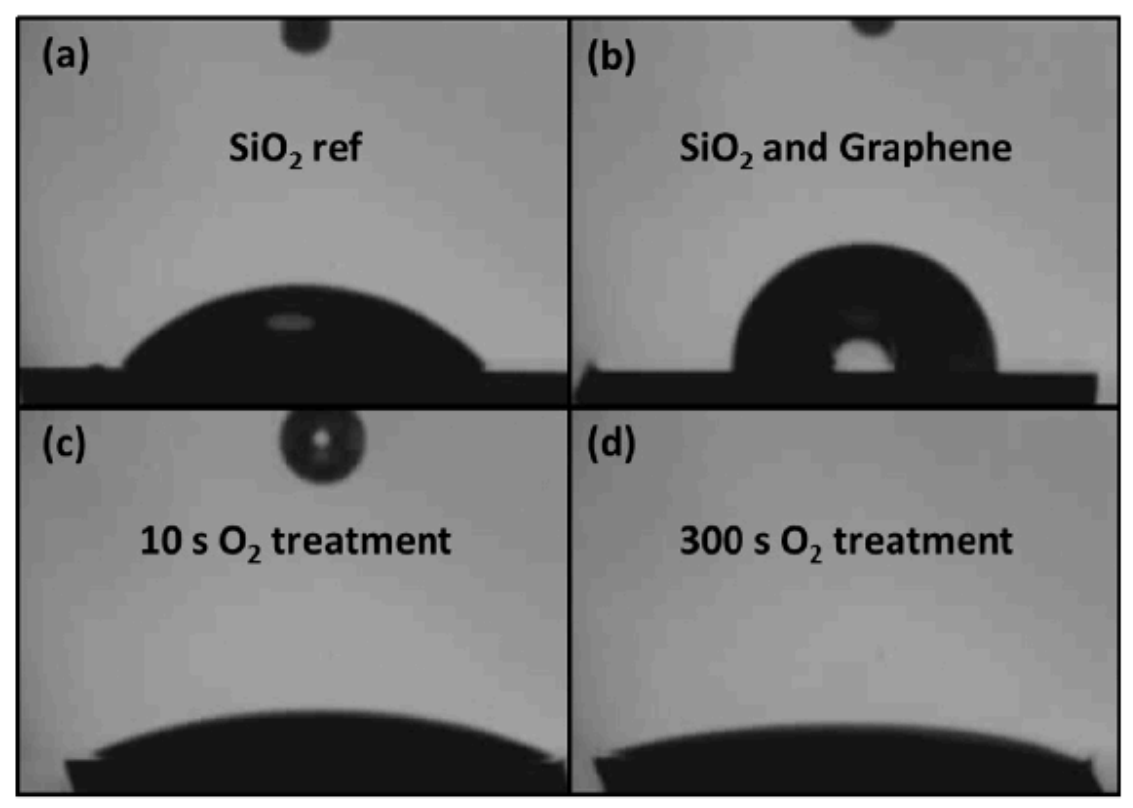

Fig. 1 - Contact angle measurements with a drop of water on (a) a reference piece of $\mathrm{SiO}_{2}, \theta_{\mathrm{c}}=56^{\circ}$ (b) as-grown graphene on $\mathrm{SiO}_{2}, \theta_{\mathrm{c}}=93^{\circ}$ (c) graphene after $10 \mathrm{~s} \mathrm{O}_{2}$ plasma treatment, $\theta_{\mathrm{c}}=31^{\circ}$ (d) graphene after $300 \mathrm{~s}$ plasma treatment, $\theta_{\mathrm{c}}=26^{\circ}$.

Raman spectroscopy also indicates the presence of increased levels of functionalities with increasing treatment time as shown in Fig. 2. The Raman spectrum of the as-grown sample shows distinct $G\left(1582 \mathrm{~cm}^{-1}\right)$ and $2 D\left(2670 \mathrm{~cm}^{-1}\right)$ bands associated with well-ordered $\mathrm{sp}^{2}$ carbon systems. The intensity ratio of the $2 \mathrm{D}$ to $\mathrm{G}$ bands is $\mathrm{I}_{2 \mathrm{D} / \mathrm{G}}=2.33$ and the $2 \mathrm{D}$ peak can be fitted with a single Lorentzian peak with a width of $31 \mathrm{~cm}^{-1}$. These factors are indicative of the presence of monolayer graphene $[5,34]$. The $D$ band $\left(1330 \mathrm{~cm}^{-1}\right)$ in graphitic systems is a defect activated breathing mode of $A_{1 g}$ symmetry [35]. Defects due to the presence of edges, vacancies and dopants give rise to this band and it can be linked with the average crystallite size in graphitic materials. In the case of our as-grown graphene sample, a very small D

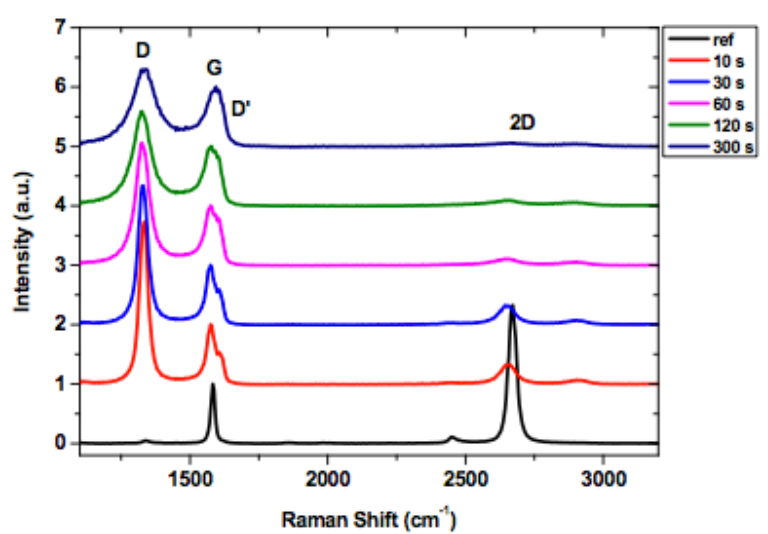

Fig. 2 - Average Raman spectra for as-grown graphene and $\mathrm{O}_{2}$ plasma treated graphene. These spectra were each obtained by averaging 6400 point spectra over a $20 \times 20 \mu \mathrm{m}$ area. band contribution is seen $\left(I_{\mathrm{D} / \mathrm{G}}=0.045\right)$ implying that the graphene is of a high quality. Furthermore, D band contributions in transferred CVD graphene can stem from defects introduced by the polymer supported transfer process [24].

The Raman spectrum undergoes a marked change following 10 s plasma treatment. A sharp and intense $D$ band is seen $\left(I_{\mathrm{D} / \mathrm{G}}=2.95\right)$ and a $\mathrm{D}^{\prime}$ peak, which is defect related and occurs via an intra-valley double resonance process in the presence of defects [29], is seen at $1610 \mathrm{~cm}^{-1}$. Both of these factors indicate an increase in defect levels or a reduction in the average crystallite size following plasma treatment. The narrow peak width of the $D$ peak $\left(\sim 35 \mathrm{~cm}^{-1}\right)$ suggests that it can be attributed to a well-defined mode, whereas the high relative integrated intensity of the $D$ to $D^{\prime}$ peak $\left(A_{D / D^{\prime}}>13\right)$ implies that the $\mathrm{D}$ peak stems from $\mathrm{sp}^{3}$ defects rather than vacancies or grain boundaries [36]. The 2D peak is suppressed ( $I_{2 D /}$ $G=0.35$ ) and broadened (width $=66 \mathrm{~cm}^{-1}$ ), which is indicative of reduced spatial uniformity or decreased crystallinity in the graphitic lattice [37]. Furthermore a D $+\mathrm{D}^{\prime}$ contribution appears at $\sim 2910 \mathrm{~cm}^{-1}$. Following an amorphisation trajectory for graphitic materials as proposed by Ferrari and Robertson [38], these observations are consistent with the transition from graphite to nanocrystalline graphite. A similar spectral change was previously reported by Ferreira et al. for mechanically exfoliated monolayer graphene bombarded with a dose of $10^{13} \mathrm{Ar}^{+}$ions per $\mathrm{cm}^{3}[39]$.

As the plasma exposure time is increased, the D and G bands broaden and after $60 \mathrm{~s}$ the $\mathrm{G}$ and $\mathrm{D}^{\prime}$ peaks start to merge and the $2 \mathrm{D}$ band intensity is further suppressed. After a plasma exposure time of $300 \mathrm{~s}$ the $\mathrm{G}$ and $\mathrm{D}^{\prime}$ bands merge and resemble a single upshifted $\mathrm{G}$ band, whereas the $2 \mathrm{D}$ band forms a broad modulated bump with surrounding bands. Such features are typically observed in heavily damaged graphitic lattices or nanocrystalline graphite $[39,40]$. 
(a)

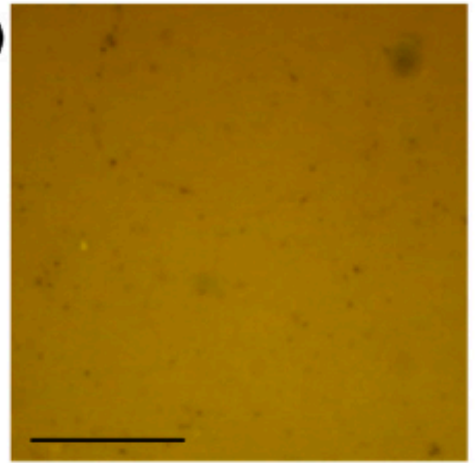

Untreated

(c)

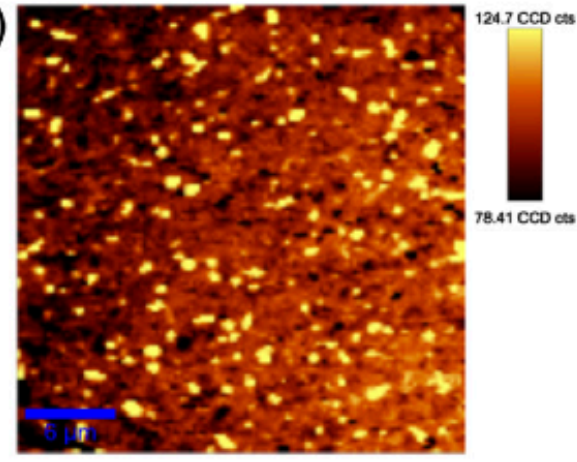

Untreated- $I_{G}$

(e)

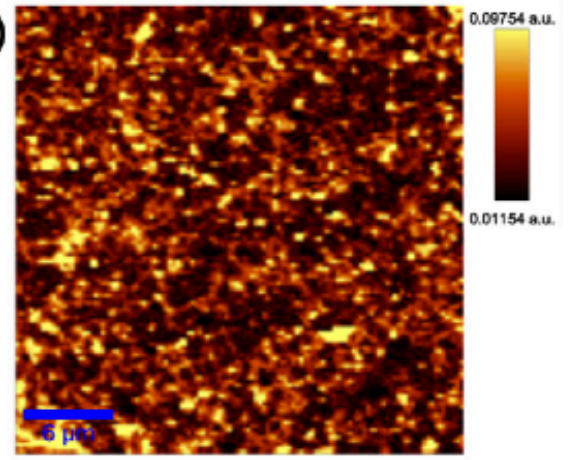

Untreated $-I_{D / G}$

(g)

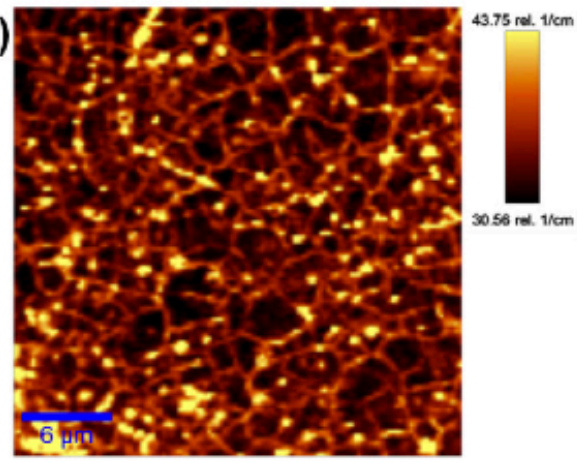

Untreated - FWHM 2D (b)

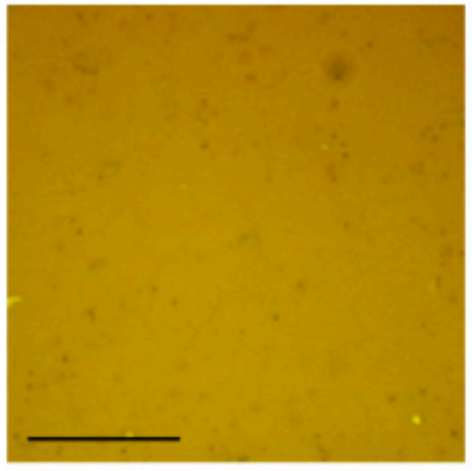

$10 \mathrm{~s} \mathrm{O}_{2}$ Plasma

(d)

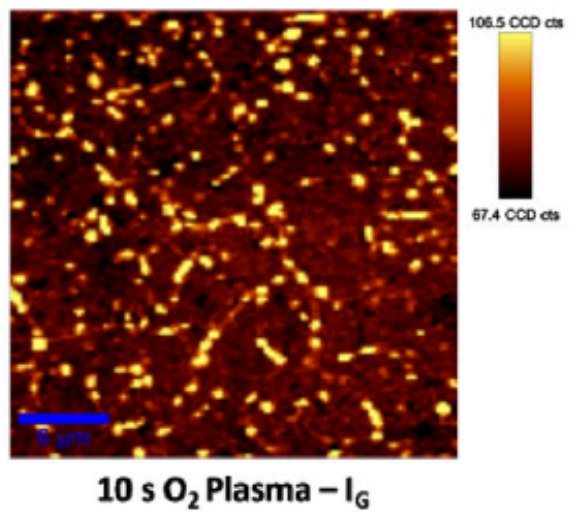

(f)

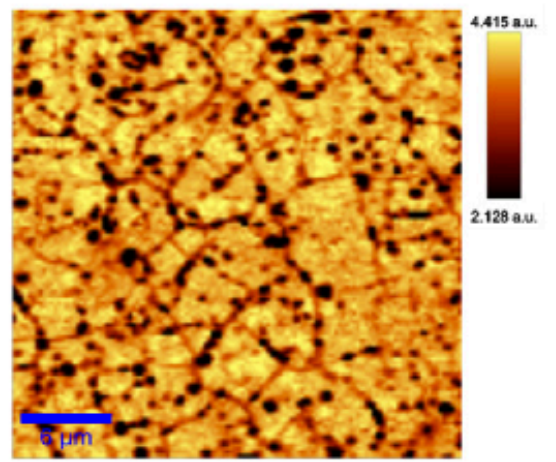

$10 \mathrm{~s} \mathrm{O}_{2}$ Plasma - $\mathrm{I}_{\mathrm{D} / \mathrm{G}}$

(h)

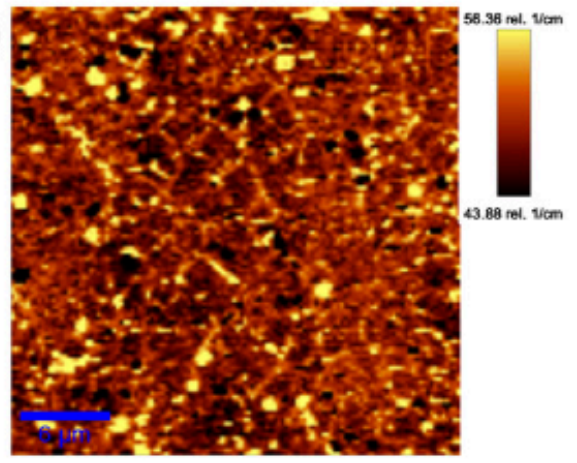

$10 \mathrm{~s}_{2}$ Plasma - FWHM 2D

Fig. 3 - (a and b) Optical microscopy images (scale bar $10 \mu \mathrm{m}$ ) and corresponding scanning Raman maps over a $30 \times 30 \mu \mathrm{m}$ area showing the ( $c$ and d) $G$ band intensity $\left(I_{G}\right)$, (e and $f$ ) the ratio of $D$ to $G$ band intensity $\left(I_{D / G}\right)$ and $(g$ and $h)$ the peak width of the 2D band. Left row: as-grown graphene. Right row: $10 \mathrm{~s}_{2}$ plasma treated graphene. 

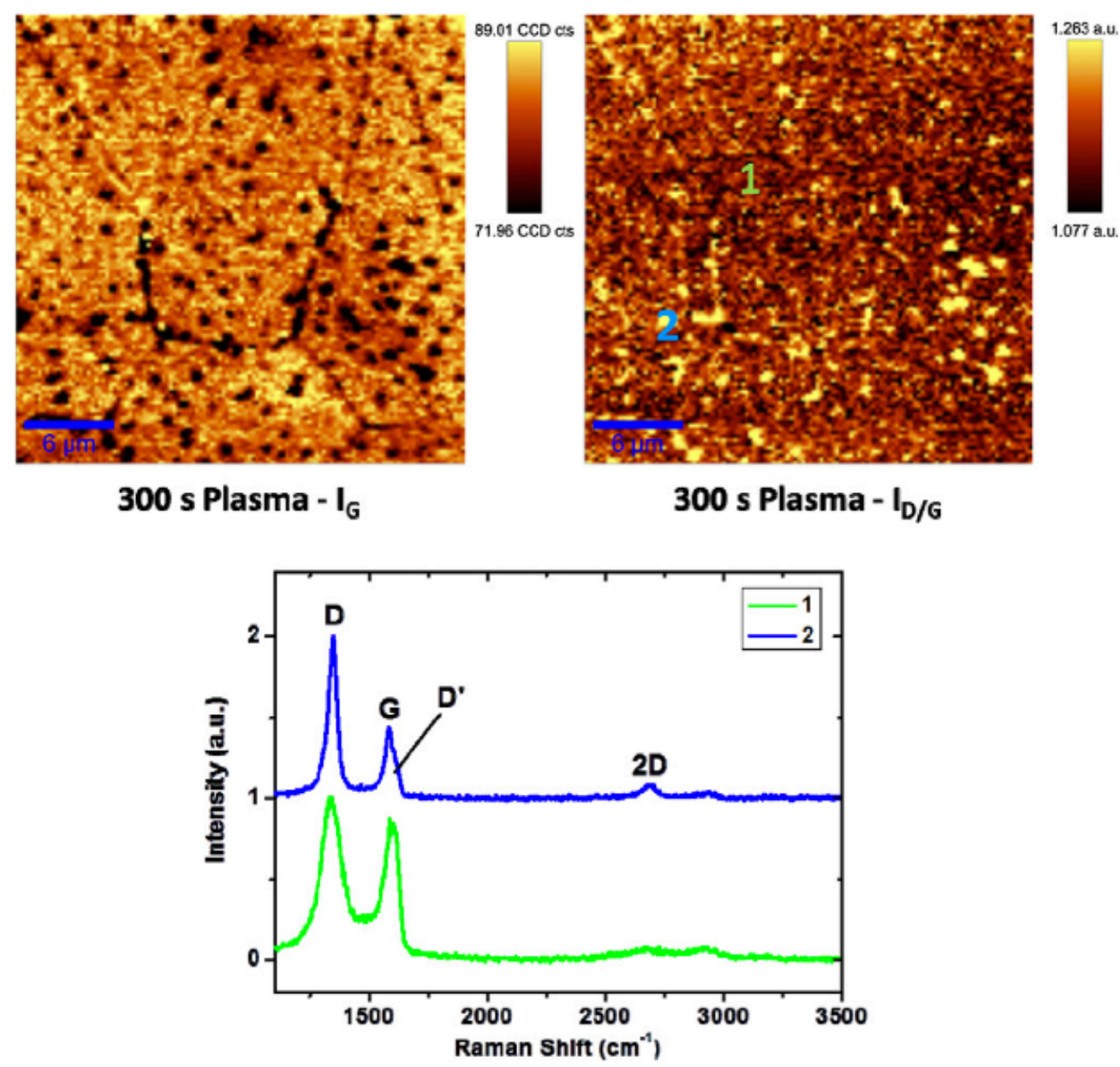

Fig. 4 - Scanning Raman maps over a $30 \times 30 \mu \mathrm{m}$ area on a $300 \mathrm{~s}$ plasma treated sample showing the $\mathrm{G}$ band intensity $\left(\mathrm{I}_{\mathrm{G}}\right)$ and the $\mathrm{D}$ to $\mathrm{G}$ band intensity ratio $\left(I_{\mathrm{D} / \mathrm{G}}\right)$ map. Bottom: Raman spectra pertaining to the marked regions on the $I_{\mathrm{D} / \mathrm{G}}$ map.

The degree of functionalisation can further be controlled by adjusting the plasma source parameters. To this end studies were carried out at a lower plasma power and also by using the plasma source in pulsed mode. Representative spectra are given in the Supporting information (Fig. S1).

Raman mapping allows for the visualisation of different bands over a large area and can give insight into the nature and defect levels of CVD graphene samples. Optical microscopy images and corresponding Raman maps for as-grown CVD graphene and $10 \mathrm{~s} \mathrm{O}_{2}$ plasma treated graphene are shown in Fig. 3. The $\mathrm{G}$ band intensity map for the as-grown graphene (Fig. 3c) indicates that there is graphitic coverage over the entire scan area. There are a number of regions of higher intensity which are consistent with secondary islands nucleating on top of the monolayer graphene giving small isolated regions of double or few layer graphene. The $\mathrm{G}$ band intensity map for the plasma treated graphene (Fig. 3d) looks similar; however, there is a greater contrast between the background graphene signal and the islands nucleating on top. $I_{D / G}$ maps for the two samples look very different due to their differing defect levels. In the case of the as-grown graphene there are spots of relative high intensity scattered across the sample (Fig. 3e), however the actual intensity of these spots is quite low $\left(I_{\mathrm{D} / \mathrm{G}}<0.1\right)$; thus implying that the graphene is of high quality. In the case of the plasma treated graphene (Fig. 3f), the majority of the scan area is seen to have a high defect level. This shows that the plasma treatment introduces defects on the surface of the graphene. In fact, the only spots with a low $\mathrm{I}_{\mathrm{D} / \mathrm{G}}$ are the areas of secondary nucleation (as seen on the $\mathrm{G}$ band intensity map). This indicates that these secondary islands are more resistant to the plasma than the monolayer background; or that they act as a mask protecting the underlying layer. The film remains continuous following plasma treatment and no additional edges, tearing or removal of graphene are seen in either optical microscopy images (Fig. 3a and b) or scanning Raman maps when compared with the as-grown sample. The peak width map for the 2D band shows a narrow peak width for most of the as-grown sample with an increase in the peak width at regions were outgrowing lateral crystallites meet each other (Fig. 3g). There is also broadening in the region of some of the secondary nucleation, but not in all of the areas suggesting a variation in the stacking mechanism from site to site. In the case of the plasma treated sample the peak width is broad $\left(>45 \mathrm{~cm}^{-1}\right)$ over the entire scan area (Fig. $3 \mathrm{~h}$ ).

Scanning Raman maps for a plasma exposure time of $300 \mathrm{~s}$ are shown in Fig. 4. The $G$ band intensity map in this case looks like an inverse of those for the as-grown and $10 \mathrm{~s}$ treated samples with low intensity spots on a higher intensity background. This is because, at this level of exposure, the G 
band and $\mathrm{D}^{\prime}$ band have merged into one broadened band. The secondary crystallites nucleating on the monolayer background are less susceptible to plasma induced defects and thus have a lower $\mathrm{D}^{\prime}$ band intensity leading to an overall lower $G$ band intensity. This is reinforced by the map of $D$ to $G$ band intensity ratio which shows dark spots corresponding to secondary islands less susceptible to plasma induced defects. Representative Raman spectra are shown inset in Fig. 4.

XPS analysis allowed for the chemical composition of asgrown and plasma treated samples to be probed. Survey scans clearly indicated an increase in the 01s peak with increasing exposure time (see Supporting information, Fig. S2). High resolution $\mathrm{C} 1 \mathrm{~s}$ peaks for each film were analysed and deconvoluted to account for the nature of the oxygen functional groups present as seen in Fig. 5. A first glance shows broadening of the C1s peak with increased plasma treatment time as well as the evolution of a shoulder peak at higher binding energies. The as-grown graphene film displays a prominent graphitic peak at $\sim 284.4 \mathrm{eV}[41-43]$ with minor contributions from other species. Defects or $\mathrm{sp}^{3}$ carbon sites are manifested as a peak at $\sim 285.5 \mathrm{eV}$, hydroxyl groups as a peak at $\sim 286.6 \mathrm{eV}$, carbonyl groups at $\sim 288 \mathrm{eV}$ and carboxyl groups at $\sim 289 \mathrm{eV}$ $[31,42-44]$. A satellite of the graphitic peak is also found at $\sim 291 \mathrm{eV}$. As expected for the as-grown graphene film (Fig. 5a), the C1s peak primarily consists of the graphitic peak with only small contributions from carbon atoms in non-graphitic environments. This is in keeping with the Raman spectroscopy data, which shows a spectrum typical of high purity monolayer graphene. The small $\mathrm{sp}^{3}$ and functional group contributions in this sample can be somewhat attributed to edgetermination of the graphene lattice.

With oxygen plasma treatment the intensity of the peaks corresponding to oxygenated functional groups relative to the graphitic peak increases, as can be seen in Fig. $5 \mathrm{~b}$ and c. These show that the oxygen plasma treatment leads to the incorporation of oxygenated functionalities on the surface of the graphene and, in turn, shows that defect contributions in the Raman spectra arise from the presence of such functional groups and not just from edges and vacancies. Of note, also, is the increase in prominence of the $\mathrm{sp}^{3} /$ defect peak at $285.5 \mathrm{eV}$ with increasing treatment time. As oxygen radicals react with the graphene film, the $\mathrm{sp}^{2}$ symmetry is destroyed and more $\mathrm{sp}^{3}$ sites emerge; which corresponds to the increased level of oxygenated functional groups.

Simple electrochemical measurements show a massive change in the properties of graphene films following plasma treatment. The behaviour of as-grown and $10 \mathrm{~s} \mathrm{O}_{2}$ plasma etched graphene in background electrolyte $(1 \mathrm{M} \mathrm{KCl})$ is shown in cyclic voltammograms in Fig. 6 . It is evident that the charging current is drastically reduced following plasma treatment; this can be attributed to the functionalisation process having an adverse effect on the conductivity of the film. In the case of CVD graphene, all the atoms are surface atoms and, consequently, surface modification will alter the conductivity of the whole film. The tilt observed in the voltammograms can be attributed to an ohmic drop which can be linked with poor contacts. Sputtered gold $(50 \mathrm{~nm})$ was used as the contact material; however, when dealing with monolayers, forming good contacts is a non-trivial affair and further work would need to be undertaken to optimise this. It is, nevertheless, evi-
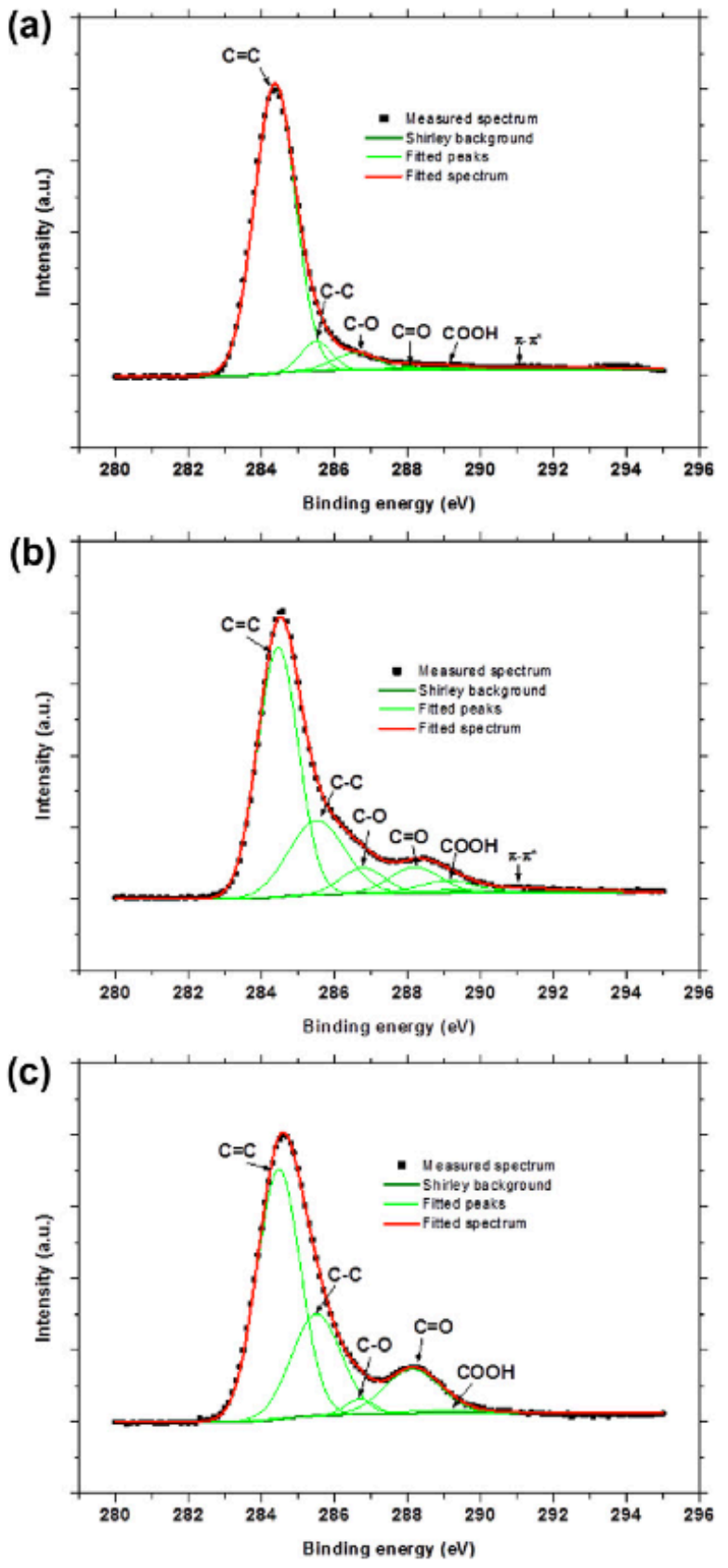

Fig. 5 - High resolution $C_{1 s}$ peak scans for (a) As-grown graphene (b) $10 \mathrm{~s} \mathrm{O}_{2}$ plasma treated graphene and (c) $300 \mathrm{~s}$ $\mathrm{O}_{2}$ plasma treated graphene.

dent that plasma exposure degrades the film's electronic properties; such plasma treatments could be useful for lithographically defining active areas on graphene-based devices.

The use of downstream oxygen plasma treatments to functionalise graphene in a controlled manner has been established. Ideally one would want such treatments to be reversible. To this end, heavily functionalised graphene ( $300 \mathrm{~s} \mathrm{O}_{2}$ plasma) was subjected to a second plasma treatment in a reducing atmosphere of $\mathrm{H}_{2}$ and $\mathrm{NH}_{3}$. A Raman spectrum of such a sample along with reference spectra from as-grown and $\mathrm{O}_{2}$ functionalised graphene is shown in Fig. 7a. It is evi- 


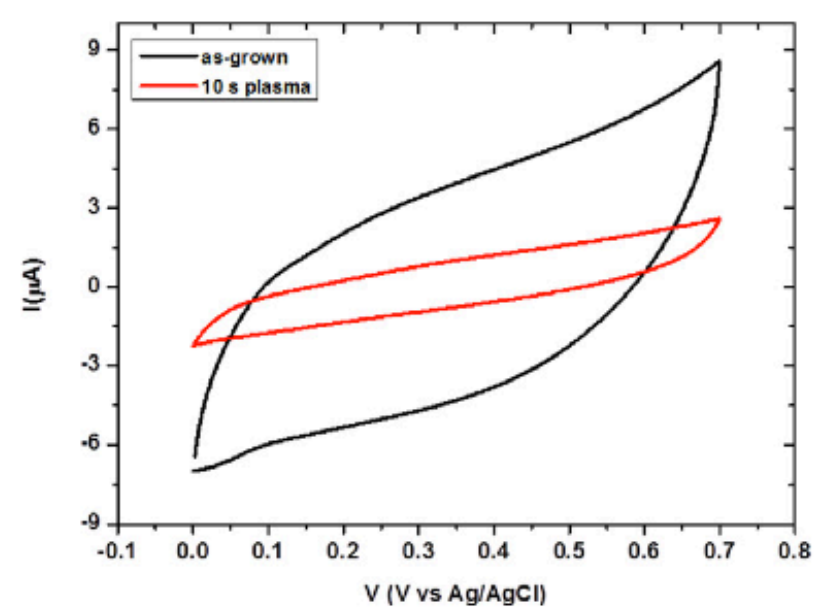

Fig. 6 - Cyclic voltammogram taken at a scan rate of $100 \mathrm{mV} /$ $\mathrm{s}$ for an as-grown and a $10 \mathrm{~s}$ plasma treated graphene sample in $1 \mathrm{M} \mathrm{KCl}$.
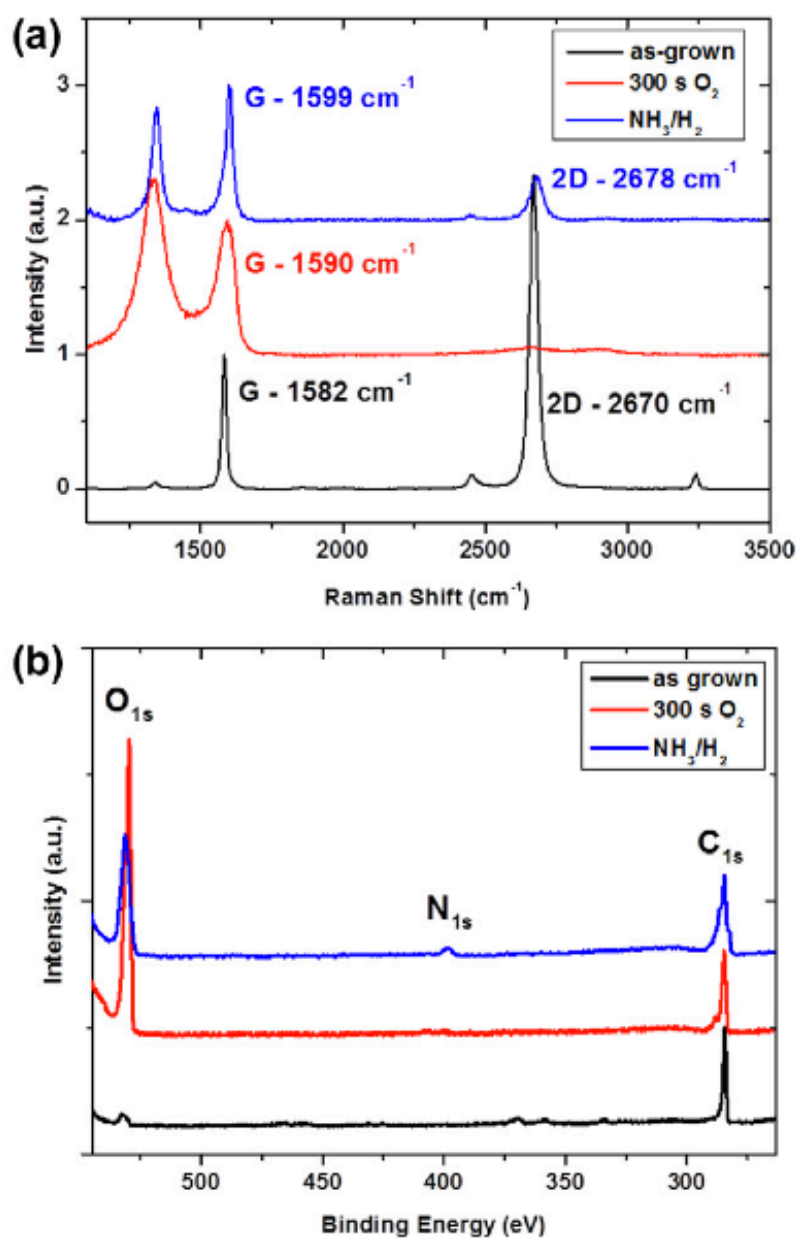

Fig. 7 - (a) Raman spectra showing as-grown graphene, $\mathrm{O}_{2}$ plasma treated graphene and graphene with a second plasma treatment in a $\mathrm{NH}_{3} / \mathrm{H}_{2}$ mixture at $500 \mathrm{~W}$. (b) Corresponding XPS survey scan illustrating a reduction in oxygen content and introduction of nitrogen following the second plasma treatment. dent that following this second plasma treatment the $D$ band is significantly narrowed and reduced in intensity. Furthermore, re-emergence of a 2D band contribution is observed. A significant reduction in oxygen content was noted by XPS analysis following this secondary treatment (Fig. $7 \mathrm{~b}$ ). These factors point towards an improvement in the crystallinity, or partial reduction of the functionalised graphene. Also of note is an upshift in both the $\mathrm{G}$ and $2 \mathrm{D}$ bands which suggests $\mathrm{N}$-doping of the graphene [28]. The emergence of a N1s peak at $\sim 400 \mathrm{eV}$ in the XPS survey scan confirms incorporation of nitrogen into the graphene lattice (Fig. $7 \mathrm{~b}$ ), the intensity of this peak indicates a doping level of greater than $3 \% . \mathrm{N}$-doped graphene is a high-interest material in the fields of energy storage and conversion [16] and bio-sensing [45]; this facile approach may furnish a wealth of applications in these areas. Interestingly, when as-grown graphene is subjected to identical $\mathrm{NH}_{3} / \mathrm{H}_{2}$ plasma treatments, no major change in its Raman spectrum is observed (see Supporting information, Fig. S3).Thus, the introduction of oxygenated functionalities is key for breaking the $\mathrm{sp}^{2}$ symmetry of graphene and facilitating the addition of further functional groups.

\section{Summary}

We have outlined a non-destructive and controllable process for the functionalisation of CVD graphene films with a downstream plasma source. Using such treatments the surface properties can be modified. Raman analysis outlines increased functionalisation with increased plasma exposure time and scanning Raman analysis allowed for the nature and location of defects to be probed. XPS allowed for the contributions of different functional groups to be measured. We also demonstrate that this process is partially reversible and can be used as a stepping stone for the incorporation of other functional groups onto the surface of graphene.

\section{Acknowledgements}

This work was supported by the SFI under Contracts No. 08/ CE/I1432, PI_10/IN.1/13030 and the EU under FP7-2010-PPP Green Cars (Electrograph Co No. 266391).

\section{Appendix A. Supplementary data}

Supplementary data associated with this article can be found, in the online version, at http://dx.doi.org/10.1016/ j.carbon.2012.11.040.

\section{REFERENCES}

[1] Geim AK, Novoselov KS. The rise of graphene. Nat Mater 2007;6(3):183-91.

[2] Novoselov KS, Geim AK, Morozov SV, Jiang D, Zhang Y, Dubonos SV, et al. Electric field effect in atomically thin carbon films. Science 2004;306(5696):666-9.

[3] Neto AHC, Guinea F, Peres NMR, Novoselov KS, Geim AK. The electronic properties of graphene. Rev Mod Phys 2009;81(1):109. 
[4] Bae S, Kim H, Lee Y, Xu X, Park J-S, Zheng Y, et al. Roll-to-roll production of 30-inch graphene films for transparent electrodes. Nat Nanotechnol 2010;5(8):574-8.

[5] Li X, Cai W, An J, Kim S, Nah J, Yang D, et al. Large-area synthesis of high-quality and uniform graphene films on copper foils. Science 2009;324(5932):1312-4.

[6] Blake P, Brimicombe PD, Nair RR, Booth TJ, Jiang D, Schedin F, et al. Graphene-based liquid crystal device. Nano Lett 2008;8(6):1704-8.

[7] Cai WW, Zhu YW, Li XS, Piner RD, Ruoff RS. Large area fewlayer graphene/graphite films as transparent thin conducting electrodes. Appl Phys Lett 2009;95(12).

[8] Park J, Lee WH, Huh S, Sim SH, Kim SB, Cho K, et al. Workfunction engineering of graphene electrodes by selfassembled monolayers for high-performance organic fieldeffect transistors. J Phys Chem Lett 2011;2(8):841-5.

[9] Strong V, Dubin S, El-Kady MF, Lech A, Wang Y, Weiller BH, et al. Patterning and electronic tuning of laser scribed graphene for flexible all-carbon devices. ACS Nano 2012;6(2):1395-403.

[10] Yim C, McEvoy N, Rezvani E, Kumar S, Duesberg GS. Carbonsilicon schottky barrier diodes. Small 2012;8(9):1360-4.

[11] Keeley GP, O'Neill A, McEvoy N, Peltekis N, Coleman JN, Duesberg GS. Electrochemical ascorbic acid sensor based on DMF-exfoliated graphene. J Mater Chem 2010;20(36):7864-9.

[12] Rumyantsev S, Liu G, Shur MS, Potyrailo RA, Balandin AA. Selective gas sensing with a single pristine graphene transistor. Nano Lett 2012;12(5):2294-8.

[13] Schedin F, Geim AK, Morozov SV, Hill EW, Blake P, Katsnelson MI, et al. Detection of individual gas molecules adsorbed on graphene. Nat Mater 2007;6(9):652-5.

[14] Wang Y, Shi ZQ, Huang Y, Ma YF, Wang CY, Chen MM, et al. Supercapacitor devices based on graphene materials. J Phys Chem C 2009;113(30):13103-7.

[15] Miller JR, Outlaw RA, Holloway BC. Graphene double-layer capacitor with ac line-filtering performance. Science 2010;329(5999):1637-9.

[16] Shao Y, Zhang S, Engelhard MH, Li G, Shao G, Wang Y, et al. Nitrogen-doped graphene and its electrochemical applications. J Mater Chem 2010;20(35):7491-6.

[17] Sun L, Wang L, Tian C, Tan T, Xie Y, Shi K, et al. Nitrogendoped graphene with high nitrogen level via a one-step hydrothermal reaction of graphene oxide with urea for superior capacitive energy storage. RSC Adv 2012;2(10):4498-506.

[18] Liston EM, Martinu L, Wertheimer MR. Plasma surface modification of polymers for improved adhesion - a critical review. J Adhes Sci Technol 1993;7(10):1091-127.

[19] Felten A, Bittencourt C, Pireaux JJ, Van Lier G, Charlier JC. Radio-frequency plasma functionalization of carbon nanotubes surface $\mathrm{O}-2, \mathrm{NH}_{3}$, and CF4 treatments. J Appl Phys 2005;98(7).

[20] Xu T, Yang J, Liu J, Fu Q. Surface modification of multi-walled carbon nanotubes by $\mathrm{O}_{2}$ plasma. Appl Surf Sci 2007;253(22):8945-51.

[21] Jones JG, Waite AR, Muratore C, Voevodin AA. Nitrogen and hydrogen plasma treatments of multiwalled carbon nanotubes. J Vac Sci Technol B 2008;26(3):995-1000.

[22] Keeley GP, McEvoy N, Kumar S, Peltekis N, Mausser M, Duesberg GS. Thin film pyrolytic carbon electrodes: a new class of carbon electrode for electroanalytical sensing applications. Electrochem Commun 2010;12(8):1034-6.

[23] McEvoy N, Peltekis N, Kumar S, Rezvani E, Nolan H, Keeley GP, et al. Synthesis and analysis of thin conducting pyrolytic carbon films. Carbon 2012;50(3):1216-26.

[24] Peltekis N, Kumar S, McEvoy N, Lee K, Weidlich A, Duesberg GS. The effect of downstream plasma treatments on graphene surfaces. Carbon 2012;50(2):395-403.
[25] Hazra KS, Rafiee J, Rafiee MA, Mathur A, Roy SS, McLauhglin J, et al. Thinning of multilayer graphene to monolayer graphene in a plasma environment. Nanotechnology 2011;22(2):025704.

[26] Xie LM, Jiao LY, Dai HJ. Selective etching of graphene edges by hydrogen plasma. J Am Chem Soc 2010;132(42):14751-3.

[27] Gokus T, Nair RR, Bonetti A, Bohmler M, Lombardo A, Novoselov KS, et al. Making graphene luminescent by oxygen plasma treatment. ACS Nano 2009;3(12):3963-8.

[28] Lin YC, Lin CY, Chiu PW. Controllable graphene N-doping with ammonia plasma. Appl Phys Lett 2010;96(13).

[29] Elias DC, Nair RR, Mohiuddin TMG, Morozov SV, Blake P, Halsall MP, et al. Control of graphene's properties by reversible hydrogenation: evidence for graphane. Science 2009;323(5914):610-3.

[30] Xie X, Qu L, Zhou C, Li Y, Zhu J, Bai H, et al. An asymmetrically surface-modified graphene film electrochemical actuator. ACS Nano 2010;4(10):6050-4.

[31] Liu L, Xie D, Wu M, Yang X, Xu Z, Wang W, et al. Controlled oxidative functionalization of monolayer graphene by watervapor plasma etching. Carbon 2012;50(8):3039-44.

[32] Kumar S, McEvoy N, Kim H-Y, Lee K, Peltekis N, Rezvani E, et al. CVD growth and processing of graphene for electronic applications. Phys Status Solidi (b) 2011;248(11):2604-8.

[33] Shin YJ, Wang Y, Huang H, Kalon G, Wee ATS, Shen Z, et al. Surface-energy engineering of graphene. Langmuir 2010;26(6):3798-802.

[34] Ferrari AC, Meyer JC, Scardaci V, Casiraghi C, Lazzeri M, Mauri F, et al. Raman spectrum of graphene and graphene layers. Phys Rev Lett 2006;97(18):187401.

[35] Tuinstra F, Koenig JL. Raman spectrum of graphite. J Chem Phys 1970;53(3):1126-30.

[36] Eckmann A, Felten A, Mishchenko A, Britnell L, Krupke R, Novoselov KS, et al. Probing the nature of defects in graphene by Raman spectroscopy. Nano Lett 2012;12(8):3925-30.

[37] Lee Y-J. The second order Raman spectroscopy in carbon crystallinity. J Nucl Mater 2004;325(2-3):174-9.

[38] Ferrari AC, Robertson J. Interpretation of Raman spectra of disordered and amorphous carbon. Phys Rev B 2000;61(20): 14095.

[39] Martins Ferreira EH, Moutinho MVO, Stavale F, Lucchese MM, Capaz RB, Achete CA, et al. Evolution of the Raman spectra from single-, few-, and many-layer graphene with increasing disorder. Phys Rev B 2010;82(12):125429.

[40] Cancado LG, Jorio A, Ferreira EHM, Stavale F, Achete CA, Capaz RB, et al. Quantifying defects in graphene via Raman spectroscopy at different excitation energies. Nano Lett 2011;11(8):3190-6.

[41] Hernandez Y, Nicolosi V, Lotya M, Blighe FM, Sun ZY, De S, et al. High-yield production of graphene by liquid-phase exfoliation of graphite. Nat Nanotechnol 2008;3(9):563-8.

[42] Poh HL, Sanek F, Ambrosi A, Zhao G, Sofer Z, Pumera M. Graphenes prepared by Staudenmaier, Hofmann and Hummers methods with consequent thermal exfoliation exhibit very different electrochemical properties. Nanoscale 2012;4(11):3515-22.

[43] Stankovich S, Dikin DA, Piner RD, Kohlhaas KA, Kleinhammes A, Jia Y, et al. Synthesis of graphene-based nanosheets via chemical reduction of exfoliated graphite oxide. Carbon 2007;45(7):1558-65.

[44] Mei X, Ouyang J. Ultrasonication-assisted ultrafast reduction of graphene oxide by zinc powder at room temperature. Carbon 2011;49(15):5389-97.

[45] Wang Y, Shao Y, Matson DW, Li J, Lin Y. Nitrogen-doped graphene and its application in electrochemical biosensing. ACS Nano 2010;4(4):1790-8. 